\title{
Resonances in Scattering. New Phenomena in Quantum Mechanics.
}

\author{
S.Pozdneev \\ P.N.Lebedev Physical Institute, Russian Academy of Sciences \\ Moscow, Russia 107924 \\ Email: pozdneev@sci.lebedev.ru
}

\begin{abstract}
The main features of resonance in scattering are described and resonances are determined on the basis of the theory of collisions in a two-body system, as well as resonances emerging as a result of collisions in a few-body system. Regularities in the emergence of such resonances and their characteristics are analyzed. The results of calculations of these resonant processes occurring during collisions of electrons with diatomic molecules, made on the basis of the quantum theory of scattering in a few-body system, based on Faddeev-Yakubovsky equations are discussed.
\end{abstract}

Keywords: Resonances in scattering, Faddeev equations, Efimov states.

\section{Introduction}

Resonances offering a variety and peculiarities of physical patterns of phenomena exist in any branch of physics from molecular physics to elementary particle physics. The concept of resonance is one of fundamental concepts in quantum physics. We can attach a broad physical meaning to the term resonance, including stable levels and implying their effect on scattering processes $[1,2]$.

Resonances play a special role in the physics of irreversible processes. In this case, in accordance with the Poincare theorem [3], resonances are responsible for nonintegrability of most dynamic systems. A theoretical explanation of resonances and their parameters can be constructed on the basis of forces of interaction between particles that are treated as elementary particles in such processes. For example, resonant processes in atomic physics are determined by the forces of interaction between electrons and nuclei, while resonances in nuclear physics are determined by forces acting between nucleons. A resonance in scattering is any peak on the experimental curve describing the scattering cross section. The resonance is characterized by the moment, parity, spin, lifetime, etc. Collisions of electrons with molecules often result in the formation of metastable negative molecular ions, which are also traditionally referred to as molecular resonances [4-11]. In this case, since atoms move slowly as compared to electrons, the electron + molecule system can be regarded as a quasi-molecule whose electron shell at each instant corresponds to a quasistationary state of such a quasimole-cule. This is in accordance with the well-known adia-batic approximation in quantum mechanics. In this approximation, various electron transitions (excitation, ionization, charge transfer) are hampered for collisions of electrons, atoms, or ions with molecules under ordinary conditions. The necessary condition for such a charge transfer $[5,6]$ is $\Delta E \Delta \tau \sim \hbar$, where $\Delta E$ is the change in the quasimolecule energy and $\Delta \tau$ is the collision time. Thus, for slow collisions, when the value of $\Delta \tau$ is large, transitions can occur only if $\Delta E$ is small; i.e., two states ofthe quasimolecule before and after the collision must be close and such a process can also be treated as a resonant process. Such a treatment of a resonance reveals the relation between equilibrium and dynamics on the one hand and the physics of dissipative processes on the other hand [12].

The importance of resonant processes is determined by the fact that all practical applications of experimental studies are based on resonances since it is resonant processes that are characterized by large cross sections or long lifetimes as compared to nonresonant processes and play an important role in low-temperature plasmas (resonant processes determine the emergence and disappearance of excited and charged particles, i.e., determine optical and electrical properties of a plasma), in controlled thermonuclear synthesis, mu-catalysis, and so on [4-12].

For example, in laser physics, molecular reactions, which produce the excited molecules, can be effectively used in chemical lasers if they have a number of the following salient features [13-15]: 
1. the thermal effect and rate constants in these reactions have to be high enough and

2. a considerable part of the energy released must transver to the energy of reaction products.

It is not surprising, then that the practical demands for extending the spectral range and increasing the power of chemical lasers have simulated and active search for such chemical reactions which can be described accurately and completely on a basis of experimental investigations. Therefore, at present theoretical methods of investigation of elementary processes are attracting considerable attention [5-11,13$15]$

Proceeding from the theory of collisions in a two-body system in which the target molecule is regarded as a force center, the following type of resonances can be distinguished [4-11].

1. A form resonance appears in the case when the impinging electron is trapped to a quasi-stationary level separated from the level in the continuum by a centrifugal barrier formed by a combination of attractive and repulsive fields of the target molecule. This type of resonance appears only when the electron possesses an angular momentum relative to the target molecule. In the case of low-energy s scattering $(l=0)$, electron cannot be trapped and form resonance is absent.

2. A vibrationally excited resonance appears when the impinging electron excites vibrations of the target molecule and is temporally bound. In this case, the kinetic energy of the electron is directly transformed into the vibrational energy of motion of the nuclei of the negative molecular ion; thus, this type of resonance is associated with violation of the Born-Oppenheimer principle. The lifetimes of such resonant states are extremely long (especially for polyatomic molecules) and attain tens of microseconds.

3. An electron-excited resonance is formed when the projectile electron excites the electron system of the target molecule and also becomes temporally bound. In this case, the detachment of an electron is impossible as long as the molecule remains in the excited state. Nevertheless, an electron still may be detached if closed and open channels are coupled.

Theoretical description of such resonances appearing as a result of formation of negative metastable ions is presented in [4-11] on the basis of the theory of scattering in a two-body system. In these works, resonances are defined as complex poles of the scattering matrix 1 continued to the non-physical energy sheet or as poles of an analytic continuation of the Green function. Collisions between electrons and molecules occurring without the formation of intermediate complexes as well as collision processes at thermal energies of impinging electrons, in which a nonmonotonic energy dependence of scattering cross section is also observed, remain unstudied. In the latter case, the application of standard techniques for calculating cross sections is unjustified in view of violation of the Born-Oppenheimer approximation [4-6]. The application of the theory of collisions in a two-body system for calculating such processes encounters considerable difficulties since the system considered here is essentially a many-particle system [13, 14].

For this reason, we will describe resonant processes occurring during collisions of an electron with molecules by using a more consistent approach based on the quantum theory of scattering in a few-particle system $[13,14]$. The main approximation in this case is that the interaction of the projectile electron with the electrons and nuclei of the target molecule is replaced by the interaction of the electrons with the atoms of the molecule, the atoms being treated as force centers. Thus, a complex many-particle system consisting of the electron and the nuclei is replaced by a system of few interacting bodies, which can be described with the help of Faddeev equations [13]. Naturally, this approximation imposes certain constraints on the energy of the projectile electron: this energy should not be higher than that the ionization energy of the atoms constituting the molecule. However, it is precisely this energy range that is interesting in connection with the presence of resonance peaks in the effective cross sections of collisions of electrons with various molecules [4-11].

In such a formalism, a resonance in a three-particle system is determined by two-particle resonances under certain conditions $[1,13,14]$. Thus, the reason for the emergence of three-particle resonances is the existence of resonant states in paired subsystems. This not very popular point of view is due to the fact that such a coupling does not exist always and cannot be determined explicitly even when it is present. This was demonstrated for the first time in nuclear physics and in elementary particle physics where the interaction between particles leading to the existence of resonances is determined by the exchange between the particles of the same resonances; thus, resonances produce themselves $[1,2]$.

In atomic physics, coupling between resonances is observed for a large number of phenomena (such as scattering of electrons by molecules, coupling between clusters in biopolymer molecules, and in Bose condensate) $[4-7,14,15]$. In this type of coupling, two-particle resonances lead to a series of three-particle 
resonance. A peculiar feature of this phenomenon is that the stronger the two-particle resonance, the larger the number of three-particle resonances produced by it. Experiments show [1, 14, 15] that such resonant states in many-particle systems lead to anomalously high rates of chemical reactions, dynamic coupling of noninteracting particles, etc. [14-16]. The importance of studying such states is directly associated with determining the binding energy of a system of $N$ bodies using information on subsystems of this many-particle system, i.e., the construction of dependences $E_{N}=f\left(E_{N-1}, E_{N-2}, \ldots\right)$ and the determination of the conditions for the formation of a coupled many-particle system provided that some subsystems are not coupled [16].

The physical foundation of the effect considered here is presented in [1], where the following aspects are revealed.

1. The effect of two-particle resonances on the spectrum of a three-particle system is clearly manifested; i.e., a two-particle resonance can radically reconstruct the discrete spectrum of three particles. However, not every two-particle resonant state can reconstruct the spectrum of three particles, but only the state whose size $r_{r e s} \sim\left(2 m_{i j}\left|e_{0}\right|\right)^{1 / 2}$ is much larger than the range $r_{0}$ of its action $\left(e_{0}\right.$ is the binding energy and $m_{i j}$ is the reduced mass of a pair of particles. Such a resonance can only be an s resonance $(l=0)$ since such resonant states strongly differ in size from other types of resonant states. For $e_{0} \rightarrow 0$, size $r_{\text {res }} \rightarrow \infty$ . The size of a resonant state is manifested in the scattering of particles in the form of a large scattering length $a$, which is equal to the size of this resonant state for small $e_{0}$. Analyzing resonant states from the standpoint of their size, we can observe that all these states sharply differ from the resonance considered above. For example, the state occupied by the system in a partial wave with $l \neq 0$ has a size on the order of the range of forces due to the centrifugal barrier; a compound resonance is not large either. Thus, a two-particle s level with a small binding energy occupies an exceptional position among resonant states as regards its size.

2. Three-particle levels are stable and their number is proportional to $\ln (a / r)$. It can be proved [1, 13-15] that the interaction responsible for the emergence of these levels has the form $U \sim A / R^{2}$, where $R^{2}=2 / 3\left(r_{1}^{2}+r_{2}^{2}+r_{3}^{2}\right), r_{i}$ is the distance between a pair of particle, and is operative in the interval $\left(r_{0}, a\right)$ (Fig. 1). In the general case, the constant $A$ of this interaction is a function of quantum numbers of the three-particle state, angular momentum, parity, and symmetry relative to the transposition of the particles. The value of $A$ is estimated in $[1,14,15]$. The strongest attraction should be observed for the orbital angular momentum $L=0$ for three particles since centrifugal forces are absent in this case. The symmetry of this state must be maximal; otherwise, the wave function has nodes and the coupling becomes weaker.

3. Centrifugal forces suppress the effect.

4. Such states possess the maximal symmetry.

5. Triple and many particles forces do not influence the effect.

6. The addition of a particle to the three-particle system suppresses the effect.

7. The particle charge has no influence on the effect which is manifested less clearly in this case.

8. For particles with spins, the effect is also pronounced less clearly.

It should be noted that such peculiar states of three particles are independent of the specific form of the potential (i.e., independent of the forces of interaction between particles) and are universal in the sense that these states reflect only the fact of existence of a resonance. Thus, irrespective of the form of pair forces between the particles, if it leads to a low-energy two-particle s resonance, this automatically leads to the formation of a family of three-particle resonances. Consequently, the reason for the emergence of three-particle level lies in the production of long-range interaction between three particles by a two-particle resonance with a large spatial size. Thus, the number of resonant states in a three-particle system is determined only by specific properties of paired subsystems. The masses of the particles have the strongest influence on the effect. The following three characteristic regimes can be singled out: the mode of identical particles, the mode of a heavy center, and the molecular mode [1, 13-15]. The heavy-center mode takes place when the masses of two particles are of the same order ml, while the mass mh of the third particle is much larger. The pair of light particle has no energy level and these particles do not interact with each other, but interact with the heavy particle through the attracting potential. In this case, if the mass of the third particle is infinitely large, we are dealing with the case of a pair of particles in a force center; naturally, three-particle levels do not emerge in such a system. In this case, the heavy particle does not respond to the motion of the noninteracting particles moving independently from each other in the field 
of the stationary heavy particle. Consequently, in this limit, the binding energy of the three particles is the additive sum of the binding energies of two-particle systems. However, for a finite mass of the heavy particle, the motion of all the three particles is correlated, so that the center of mass of the system remains at rest. In this case, the heavy particle responds to a change in the position of other particles whose motion becomes correlated in spite of the absence of a direct interaction between them. Thus, dynamic correlation in the motion of coupled particles can be treated as a sort of attraction. It should be noted that such a dynamic attraction also appears in the case when repulsive force act between the particles coupled in this way. In this case, dynamic attraction compensates mutual repulsion and leads to stabilization of the system. This can be clearly seen, for example, for the ion of positronium $e^{+} e^{-} e^{-}$ [14-16]. In this case, for any finite mass of a heavy center, the number of levels is

$$
N \sim \frac{m_{l}}{m_{h}} \ln \frac{1}{e_{0} m_{l} r_{0}^{2}}
$$

A special feature of this mode is that extremely shallow levels in paired subsystem are required for the existence of three-particle levels in contrast to the molecular mode, where the requirements imposed on paired levels are much less stringent and more realistic.

In the molecular mode, when a light particle has shallow levels in the interaction with the heavy particles, the number of levels is

$$
N \sim \sqrt{\frac{m_{l}}{m_{h}}} \ln \frac{1}{\left|e_{0}\right| m_{l} r_{0}^{2}}
$$

and the potential of the interaction produced by the light particle has the form

$$
V \sim \frac{-0.32}{m_{l} r_{h h}^{2}}
$$

which is precisely the energy of the molecular energy level. The simple example of this mode is a system consisting of an electron and two neutral atoms. The molecule formed in this way differs from a conventional molecule in that its nuclei vibrate in region $\mathrm{R}$ whose size is determined by the energy e0 of the shallow paired level; in addition to vibrational levels, this system also has a rotational spectrum. Thus, two-particle levels in this mode lead to the formation of a series of not only vibrational, but also rotational levels $[1,13-15]$. It should be noted that such peculiar resonance states are manifested in a wide range of conditions and form a stable phenomenon which can be reliably identified and confirmed experimentally.

\section{Basic Equations and Main Approximation}

We will analyze these peculiar resonant states quantitatively in the case of the molecular mode using the Faddeev integral equations [13]. In the given approximation (three particles, viz., two atoms and an electron), these equations are formulated for three parts into which the total wave function of the three-body system splits,

$$
\Psi=\sum_{i=1}^{3} \Psi_{i}
$$

Each part corresponds to possible divisions of the system of three particles into noninterecting subgroups. In the momentum space, in the case of scattering of particle 1 from the coupled pair $(2,3)$ the equations have the form $[13,14]$

$$
\Psi_{i}=\Phi_{i} \delta_{i 1}-G_{0}(Z) T_{i}\left(\Psi_{j}+\Psi_{k}\right), \quad i, j, k=1,2,3 ; 3,1,2 ; 2,1,3 ;
$$

Here, $\Phi_{1}$ describes the initial state of the three body systems: free motion particle 1 and the bound state of pair $(2,3) ; G_{0}(Z)=\left(H_{0}-Z\right)^{-1}, Z=E+i 0$, where $H_{0}$ is the operator of free motion of three particles; $E$ is the total energy of three-body system, which is equal to the sum of kinetic energy of projectile 1 and the binding energy of pair $(2,3) ; T_{i}$ is a paired T-matrix that can be unambigously defined in terms of the paired interaction potential $V_{i}$ with the help of the Lippmann-Schwinger equations

$$
T_{i}=V_{i}+V_{i} G_{i} T_{i}, \quad G_{i}=\left(h_{i}-Z_{i}\right)^{-1}, \quad h_{i}=\Delta_{i}+V_{i}
$$


To describe the motion of three particles in center-of-mass system we use the generally accepted Jacobi coordinates. It should be borne in mind that we must use as integration variables in Eq. (1) a certain system of variables which is found to be most convenient. For example, in the integral corresponding to the expression $G_{0} T_{1} \Psi_{2}$, it is more convenient to take $\boldsymbol{k}_{2}$ and $\boldsymbol{p}_{2}$ as integration variables. In this case, variables $\boldsymbol{k}_{1}$ and $\boldsymbol{p}_{1}$ determining the kernel of operator $T_{1}$ should be expressed in terms of variables $\boldsymbol{k}_{2}$ and $\boldsymbol{p}_{2}$. Sometimes, it is more convenient to use variables $\boldsymbol{p}_{1}$ and $\boldsymbol{p}_{2}$ in the same situation.

Paired $T$ matrices $t_{i}\left(\boldsymbol{k}_{i}, k_{i}^{\prime} ; Z\right)$ appearing in the kernels of the equations have singularities in variable $\mathrm{Z}$ : the poles corresponding to the discrete spectrum of paired subsystems and a cut along the positive part of the real axis generated by the spectrum of the two-body problem. The explicit form of these singularities gives the spectral representation of matrix $T$. The poles of the $T$ matrix corresponding to the discrete spectrum generate singularities in the wave function components $\Psi_{i}$; separating these components, we obtain the representation

$$
\Psi_{i}\left(\boldsymbol{k}_{i}, \boldsymbol{p}_{i} ; \boldsymbol{p}_{i}^{o}\right)=\varphi\left(\boldsymbol{k}_{i}\right) \delta\left(\boldsymbol{p}_{i}-\boldsymbol{p}_{i}^{o}\right)-B_{i}\left(\boldsymbol{k}_{i}, \boldsymbol{p}_{i} ; \boldsymbol{p}_{i}^{o} ; Z\right) /\left(p_{i}^{2} / 2 n_{i}+k_{i}^{2} / 2 m_{j k}-Z\right),
$$

where

$$
B_{i}\left(\boldsymbol{k}_{i}, \boldsymbol{p}_{i} ; \boldsymbol{p}_{i}^{o} ; Z\right)=-\sum_{j=1}^{3}\left[Q_{j}\left(\boldsymbol{k}_{i}, \boldsymbol{p}_{i} ; \boldsymbol{p}_{i}^{o} ; Z\right)-\varphi_{j}\left(\boldsymbol{p}_{j}\right) R_{j i}\left(\boldsymbol{k}_{j} ; \boldsymbol{p}_{i}^{o} ; Z\right) /\left(p_{j}^{2} / 2 n_{j}-\kappa_{j}-Z\right)\right],
$$

and $Q_{j}, R_{j i}$ are smooth function of their variables. Such a division of singularities appears automatically in the numerical solution of integral equations. To define functions $Q_{j}$ and $R_{i j}$ unambiguously, we can proceed as follows. We substitute $\Psi_{i}$ in form (3) into initial equations (1) and equate the coefficients of identical singularities. This gives the equations for these functions which can be used for expressing explicitly all main characteristics of the three-body problem: wave function, elements of the $S$ matrix, as well as the amplitudes and cross sections of all processes occurring in the three-body system. Thus, the cross section of the elastic scattering process has the form

$$
d \sigma_{11} / d \Theta=(2 \pi)^{4} n_{1}\left|R_{11}\right|^{2},
$$

the cross section of rearrangement processes is given by

$$
d \sigma_{1 i} / d \Theta=(2 \pi)^{4} n_{i} p_{f}\left|R_{1 i}\right|^{2} / p_{1}^{0}
$$

and the cross section of the process of decay into three free particles has the form

$$
d \sigma_{1 \rightarrow 3} / d \Theta d p=(2 \pi)^{4} n_{i} p_{f}\left|B_{0 i}\right|^{2} / p_{1}^{0},
$$

where

$$
p_{f}^{2}=2 n_{i}\left(p_{i}^{02} / 2 n_{i}-\kappa_{1}^{2}-\kappa_{i}^{2}\right)
$$

The main advantage of the Faddeev equations (1) is that

(i) the solution of this equation gives simultaneously the amplitudes and cross sections of all processes occurring in the three-particle system;

(ii) the accuracy in determining the bound state from the solution of the Faddeev equations is much higher than the accuracy obtained by solving the Schrodinger equations (this peculiarity is associated with the fact that Eqs. (1) were formulated for the wave function components and, hence, take into account possible asymptotic forms of the three-particle system);

(iii) these equations make it possible to carry out a correct (from the standpoint of mathematics) analysis of scattering processes, in which all three free particles are in the initial state [12, 13]; this is impossible in all approaches proposed earlier [5-11]: 


$$
1+2+3 \rightarrow \begin{cases}1+(2,3) & \text {-elastic scattering processes } \\ 1+(2,3)^{*} & \text {-excitation processes } \\ 3+(1,2)^{*} & \text {-rearrangment processes } \\ 2+(1,3)^{*} & \text {-with excitation } \\ 1+2+3 & \text {-ionization processes }\end{cases}
$$

In this case, we have the following representation for the wave function [13-15]:

$$
\Psi_{0}\left(\boldsymbol{k}, \boldsymbol{p} ; \boldsymbol{k}^{0}, \boldsymbol{p}^{0}\right)=\delta\left(\boldsymbol{k}-\boldsymbol{k}^{0}\right) \delta\left(\boldsymbol{p}-\boldsymbol{p}^{0}\right)-\frac{\sum_{i, j} M_{i j}\left(\boldsymbol{k}, \boldsymbol{p} ; \boldsymbol{k}^{0}, \boldsymbol{p}^{0} ; \frac{k^{02}}{2 m}+\frac{p^{02}}{2 n}+i 0\right)}{\frac{p^{2}}{2 n}+\frac{k^{2}}{2 m}-\frac{k^{02}}{2 m}+\frac{p^{02}}{2 n}+i 0},
$$

where functions Mi, $\mathrm{j}$ satisfy the following system of equations:

$$
M_{i j}(Z)=\delta_{i, j} T_{i}(Z)+T_{i}(Z) G_{0}(Z) \sum_{k \neq i} M_{k j}(Z)
$$

For cross sections of these processes, we obtain the following expression $[13,15]$

$$
\begin{gathered}
S_{00}\left(\boldsymbol{k}, \boldsymbol{p} ; \boldsymbol{k}^{\prime}, \boldsymbol{p}^{\prime}\right)=\delta\left(\boldsymbol{k}-\boldsymbol{k}^{\prime}\right) \delta\left(\boldsymbol{p}-\boldsymbol{p}^{\prime}\right)-2 \pi i \delta\left(\frac{p^{2}}{2 n}+\frac{k^{2}}{2 m}-\frac{p^{\prime 2}}{2 n}-\frac{k^{\prime 2}}{2 m}\right) \\
\sum_{i, j} M_{i j}\left(\boldsymbol{k}, \boldsymbol{p} ; \boldsymbol{k}^{\prime}, \boldsymbol{p}^{\prime} ; \frac{k^{\prime 2}}{2 m}+\frac{p^{\prime 2}}{2 n}+i 0\right)
\end{gathered}
$$

corresponds to processes in which three free particles are in the initial and final states,

$$
\begin{gathered}
S_{0 s_{i}}\left(\boldsymbol{k}, \boldsymbol{p} ;, \boldsymbol{p}_{i}^{\prime}\right)=2 \pi i \delta\left(\frac{p^{2}}{2 n}+\frac{k^{2}}{2 m}+\kappa_{s_{i}}^{2}-\frac{p_{i}^{\prime 2}}{2 n_{i}}\right) \sum_{k} Q_{k i}^{s_{i}}\left(\boldsymbol{k}, \boldsymbol{p} ; \boldsymbol{p}_{i}^{\prime} ;-\kappa_{s_{i}}^{2}+\frac{p_{i}^{\prime 2}}{2 n_{i}}-i 0\right)+ \\
\sum_{s_{k}} \psi_{s_{k}}\left(\boldsymbol{k}_{k}\right) R_{k i}^{s_{k} s_{i}}\left(\boldsymbol{p} ; \boldsymbol{p}_{i}^{\prime} ;-\kappa_{s_{i}}^{2}+\frac{p_{i}^{\prime 2}}{2 n_{i}}+i 0\right), \\
S_{s_{i} 0}\left(\boldsymbol{p}_{i} ; \boldsymbol{k}^{\prime}, \boldsymbol{p}^{\prime}\right)=2 \pi i \delta\left(-\kappa_{s_{i}}^{2}+\frac{p_{i}^{\prime 2}}{2 n_{i}}-\frac{p^{\prime 2}}{2 n}-\frac{k^{\prime 2}}{2 m}\right) \sum_{j} \tilde{Q}_{j i}^{s_{i}}\left(\boldsymbol{p}_{i} ; \boldsymbol{k}^{\prime}, \boldsymbol{p}^{\prime} ; \frac{k^{\prime 2}}{2 m}+\frac{p^{\prime 2}}{2 n}+i 0\right)+ \\
\sum_{s_{j}} \psi_{s_{j}}\left(\boldsymbol{k}_{j}^{\prime}\right) R_{i j}^{s_{i} s_{j}}\left(\boldsymbol{p}_{i} ; \boldsymbol{p}_{i}^{\prime} ; \frac{k^{\prime 2}}{2 m}+\frac{p^{\prime 2}}{2 n}+i 0\right),
\end{gathered}
$$

correspond to processes in which a coupled pair of particles $s_{j}$ is present in the initial or the final state. The equations for functions $Q_{j}, Q_{j}$, and $R_{i j}$ are analogous to the equations for $M_{i j}$ and are given in [13-15]. It should be noted that potentials do not appear explicitly in integral equations (1); these equations contain a more general characteristic, viz., $T$ matrices, which are connected with the potentials of the Lippmann-Schwinger equations (2). Consequently, although potentials are formally used in the given method, we essentially model T matrices, which are constructed on the basis of the Bateman method [13, 14] suitable for any local potential. This method considerably simplifies numerical solution of the system of integral equations (1) and sometimes even leads an analytic solution [13-17].

Integral equations (1) possess good properties (from the mathematical point of view) such as the Fredholm property and unambiguous solvability only under certain conditions imposed on two-particle data [13]:

(i) paired potentials $V_{i}\left(\boldsymbol{k}, \boldsymbol{k}^{\prime}\right)$, which are nonlocal in the general case, are smooth functions of $\boldsymbol{k}, \boldsymbol{k}^{\prime}$ and satisfy the condition

$$
\left|V_{i}\left(\boldsymbol{k}, \boldsymbol{k}^{\prime}\right)\right| \leq\left(1-\left|\boldsymbol{k}-\boldsymbol{k}^{\prime}\right|\right)^{1-\epsilon}, \quad \epsilon>0
$$


(ii) point $Z=0$ is not a singular point for Eqs. (2); i.e., all three scattering lengths in pair channels are finite;

(iii) the positive two-particle spectrum is continu- ous. This condition is essential for nonlocal potentials since positive eigenvalues may appear only in this case, and this condition is satisfied virtually for all physical processes.

Coulomb potentials and hard-core potentials do not satisfy the first condition:

Coulomb potentials lead to a singularity of the type $\left|\boldsymbol{k}-\boldsymbol{k}^{\prime}\right|^{-2}$ in $T$ matrices, while hard-core potentials result in a slow decrease in the T matrix for large momenta. When the second condition is violated, the Fredholm property of Eqs. (1) is lost for $Z=0$, which leads to the above-mentioned effect of emergence of an infinitely large discrete spectrum in a three-body system under certain conditions. A similar situation emerges in the case of scattering of electrons from diatomic molecules, for which the Efimov levels were experimentally observed for the first time. The approximation considered here reproduces these experimental results in a quite natural way.

It should be emphasized once again that the given approximation appears quite reasonable for values of the impinging electron energy lower than the electron excitation energy of the molecule.

As the initial data in such a formulation of the problem, we use pair interaction potentials, masses, and energies of colliding particles. For potentials of pair interaction of electrons with atoms of the molecule, we used potentials of the form

$$
V(r)=\lambda \exp (-\beta r) / r,
$$

whose parameters were determined on the basis of the electron binding energy at a negative ion, scattering lengths, and effective radius. Allowance for spin (in the case of homonuclear molecules) was made as follows. For the scattering length, we used the quantity [5, 6, 14-17]

$$
\frac{1}{a}=\frac{1}{a_{1}}=\frac{1}{a_{2}}=\frac{1}{4}\left(\frac{3}{a_{t}}+\frac{1}{a_{s}}\right),
$$

where $a_{t}$ and $a_{s}$ are the triplet and singlet scattering lengths, respectively. Pair potentials of interaction between atoms in molecules were simulated by the Morse potentials

$$
V(r)=D\left(1-\exp \left(-\alpha\left(r-r_{0}\right)\right)\right)
$$

whose parameters were determined on the basis of spectroscopic data [18].

Numerical solution of integral equations (1) involves considerable difficulties because the kernels of integral equations (1) contain the same singularities [13-15] but here, we propose a quite universal method for solving system of equations (1) for calculating bound states as well as scattering states in systems with arbitrary masses, which interact via arbitrary pair short-lived potentials that can also be defined numerically. In the method proposed here, the domain of an unknown function is divided into a number of intervals on each of which the function is approximated with the help of corresponding interpolation polynomials. The method for solving system of equations (1) is a modification of the standard method for solving integral equations, in which the integral on the right-hand side is replaced with the help of a quadratures formula for solving Eq. (1). As a result, we arrive at a system of algebraic equations for values of the sought function at the nodes of the quadratures formula. In the proposed method, the domaine of the sought function is divided into a number of segments, on each of which the function is determined with the help of interpolation polynomials reproducing the correct behavior of the function in the vicinity of the above singularities, after which integration is carried out using quadratures formulas. A package of applied programs were used for realization of the proposed numerical method for solving system of integral equations (1) $[13,15]$.

Computational difficulties encountered in calculation of cross sections in the given approximation are mainly associated with the long-range Coulomb interaction potentials. It was mentioned above that in this case the integral Faddeev equations cannot be applied directly; either these equations should be modified, or the differential formulation of the Faddeev equations in the coordinate state should be used [13-15]. In case for three charged particles Faddeev equation in the coordinate space, which have the form $[13-15]$

$$
\left(-\Delta_{x_{i}}-\Delta_{y_{i}}+V_{i}\left(x_{i}\right)-E\right) \Psi_{i}=-V_{i} \sum_{j \neq i} \Psi_{j},
$$


where

$$
\begin{gathered}
V_{i}=n_{i} / x_{i}+V_{s t}\left(x_{i}\right), \quad n_{i}=\frac{q_{k} q_{j}}{\sqrt{2 m_{k j}}}, \\
\boldsymbol{x}_{i}=\sqrt{\frac{2 m_{j} m_{k}}{m_{j}+m_{k}}}\left(\boldsymbol{r}_{j}-\boldsymbol{r}_{k}\right), \quad \boldsymbol{y}_{i}=\sqrt{\frac{2 m_{i}\left(m_{j}+m_{k}\right)}{m_{i}+m_{j}+m_{k}}} \boldsymbol{r}_{i}-\frac{m_{j} \boldsymbol{r}_{j}+m_{k} \boldsymbol{r}_{k}}{m_{j}+m_{k}},
\end{gathered}
$$

and the coordinates are connected via the relations

$$
\begin{aligned}
& \boldsymbol{x}_{i}=c_{i j} \boldsymbol{x}_{j}+s_{i j} \boldsymbol{y}_{j}, \quad \boldsymbol{y}_{i}=-s_{i j} \boldsymbol{x}_{j}+c_{i j} \boldsymbol{y}_{j}, \\
& s_{i j}^{2}=\frac{m_{k} \sum_{k} m_{k}}{\left(m_{i}+m_{j}\right)\left(m_{j}+m_{k}\right)}, \quad s_{i j}^{2}+c_{i j}^{2}=1
\end{aligned}
$$

$V_{s t}$ being pair short-range interaction potentials defines by (4) and (5).

The relation between the momentum and coordinate representations is defined by the Fourier transformation,

$$
\Psi\left(\boldsymbol{k}_{i}, \boldsymbol{p}_{i}\right)=(2 \pi)^{-3} \int \exp -i\left(\boldsymbol{k}_{i} \boldsymbol{x}_{i}+\boldsymbol{p}_{i} \boldsymbol{y}_{i}\right) \Psi\left(\boldsymbol{x}_{i}, \boldsymbol{y}_{i}\right) d \boldsymbol{x}_{i} d \boldsymbol{y}_{i}
$$

To obtain a unique solution of integrodifferential equations in the coordinate space, we must add the boundary conditions, which have the form [13-15]

$$
\begin{gathered}
\Psi_{i}\left(\boldsymbol{x}_{i}, \boldsymbol{y}_{i}\right)_{x_{i}, y_{i} \rightarrow 0} \rightarrow 0 \\
\Psi_{i}\left(\boldsymbol{x}_{i}, \boldsymbol{y}_{i}\right)_{\rho=\sqrt{x^{2}+y^{2}} \rightarrow \infty} \rightarrow \phi_{i}\left(x_{i}\right) \exp \left(i \boldsymbol{k}_{i} \boldsymbol{y}_{i}-i w_{i}^{0}\right)+ \\
\sum_{j} A_{i j}\left(\hat{y}_{j}, \hat{k}_{i}\right) \phi_{i}\left(x_{j}\right) \frac{\exp \left(i \sqrt{E_{j}}\left|\boldsymbol{y}_{j}\right|+i w_{i j}\right)}{\left|y_{j}\right|}+A_{0 i}\left(\hat{X}, \hat{k}_{i}\right) \frac{\exp \left(i \sqrt{E}|X|+i w_{0}\right)}{|X|^{5 / 2}}
\end{gathered}
$$

where

$$
\begin{gathered}
w_{i}^{0}=\frac{n_{i}}{2\left|\boldsymbol{k}_{i}\right|} \ln \left[\left|\hat{k}_{i}\right|\left|\hat{x}_{i}\right|-\left(\boldsymbol{k}_{i}, \boldsymbol{x}_{i}\right)\right], \quad w_{i j}=\sum_{k \neq j} \frac{n_{k}}{2 \mid s_{j k} \sqrt{E_{k}}} \ln 2 \sqrt{E_{k}}\left|\boldsymbol{y}_{k}\right|, \\
w_{0}=-\frac{|\boldsymbol{X}|}{2 \sqrt{E}} \sum_{i} \frac{n_{i}}{\left|\boldsymbol{x}_{i}\right|} \ln 2 \sqrt{E}|\boldsymbol{X}|, \quad n_{i}=\frac{k q_{i} q_{j}}{\sqrt{2 m_{i j}}}, \quad E_{k}=E-\kappa_{j},
\end{gathered}
$$

A large number of various numerical methods have been developed on the basis of approximation of components $\Psi$ by bicubic Hermite splines, quintet basis splines, etc. However, an effective, reliable, and universal algorithm of numerical solution of Eqs. (6) with boundary conditions (7) and (8) in the coordinate space has not been developed for the following reasons.

First, an algorithm of numerical solution for processes with three free particles in the initial and final states does not exist in view of rather complex boundary conditions.

Second, point-by-point convergence of the obtained result to the exact solution upon a decrease in the mesh size cannot be proved analytically in any of the known numerical methods based on finite different approximation.

Consequently, the application of the mesh method in the polar coordinate system [14] for solving numerically the system of coupled integrodifferential equations (6) in partial derivatives with boundary conditions (7) and (8) appears as most justified since analytic solutions also exist in this case for some potentials determining the resonant states under investigation [13,14,22]. This makes it possible to monitor the accuracy of the solutions obtained by the numerical method.

Let us consider the geometrical (topological, spatial) characteristics of the above-mentioned peculiar resonant states. Since it is quite difficult to study these characteristics experimentally in the case of electron collisions with molecules, we will consider the systems that are accessible for experimental studies, viz., clusters of molecules of inert gases [28]. 
It should be noted that these molecular clusters consisting of atoms of helium, lithium, and a number of inert gases attract attention of both theoreticians [29] and experimentalists [28] primarily due to applied studies such as superfluidity, superconductivity, Bose condensation, chemistry and physics of clusters, laser physics (i.e., the possibility of developing $\mathrm{He}_{2}^{+}$molecular laser), as well as the possibility of observing such a peculiar quantum effect in real systems.

However, a direct theoretical analysis of even the simplest of the above systems, viz., $\mathrm{He}_{3}$ consisting of three helium nuclei and six electrons, is an extremely complicated problem.

To analyze the $\mathrm{He}_{3}$ system, we consider the cluster approximation in which this system is replaced by a simpler system consisting of three force centers (helium atoms). The validity of this approximation for calculations of bound states is obvious since the difference between the binding energy of the system and the ionization energy of the atom is several orders of magnitude. It is well known that helium atoms are bosons; consequently, the problem boils down to analysis of three pairwise identical neutral spinless particles. To solve this problem, we propose mathematically correct model-free methods in the theory of scattering in the three-body system [13-15].

It should be emphasized that virtual levels in paired subsystems in the case of complex many-particle systems do not lead to the emergence of resonant states in a many-particle system [1]. This, however, does not mean that this effect is absent in these systems since it can be due to many-particle and not two-particle virtual states.

For this reason, we will consider the interpretation of a number of peculiar properties of systems $\mathrm{He}_{3}$, $A r_{3}, K r_{3}, N e_{3}, X e_{3}, L i_{3}$, and $R n_{3}$ precisely on the basis of the three-particle approximation. It should be noted that a large number of theoretical and experimental methods exist for studying clusters consisting of atoms of helium and a number of inert gases. Most methods are intended for studying bound states; however, scattering states [28-31], which are most informative for confirming the existence of peculiar resonant states, were practically ignored.

It was stated by a number of authors [30] that the main difficulties in studying the He3 system are associated with its low binding energy $(1 \mathrm{mK})$, an unusually large size of the excited state $\left(\sim 150 A^{\circ}\right)$, and a strong repulsion at small distances. However, the results obtained in [15, 31], where an analogous three-particle approximation was used for calculating the $\mathrm{He}_{3}$ system, differ from the statements made in [30].

For this reason, it would be also interesting to verify the conclusions drawn in [30] on the basis of the three-particle approximation with the short-range pair potentials used in [32]. The main purposes of this investigation are

(i) determining the number of possible resonant states;

(ii) clarifying the role of pair interaction potentials in the characteristics of these states;

(iii) estimating the effect of repulsion at short distances, which can be approximated by a hard core in the model for the boundary conditions [13-15] imposed on the characteristics of these peculiar states.

Thus, the theoretical analysis of the $\mathrm{He}_{3}$ system is reduced to solving equations in the quantum theory of scattering in a three-body system, which makes it possible to use the well-known methods [13-15]. In contrast to [30], where resonances in a three-particle system were studied using the Faddeev equations on the basis of analytic continuation of the scattering matrix to the range of complex energy values, we are using here direct numerical solution without an analytic continuation.

In this case, after the separation of angular variables, the Faddeev equations (6) in the coordinate space for the $\mathrm{He}_{3}$ system in the three-particle approximation with pair short-range potentials [32] have the form $[13-15]$

$$
\left[H_{\lambda, l}-z\right] \Psi_{a L}(x, y)=-V(x)\left(\Psi_{a L}(x, y)+\sum_{a^{\prime}} \int_{-1}^{+1} \Psi_{a^{\prime} L}\left(x^{\prime}, y^{\prime}\right) h_{a a^{\prime}}^{L}\left(x^{\prime}, y^{\prime}, \eta\right) d \eta\right)
$$

where

$$
\begin{array}{r}
H_{\lambda, l}=-\frac{\partial^{2}}{\partial x^{2}}-\frac{\partial^{2}}{\partial y^{2}}+\frac{l(l+1)}{x^{2}}+\frac{\lambda(\lambda+1)}{y^{2}} \\
z=E+i 0, \quad L=l+\lambda, \quad a=(l, \lambda),
\end{array}
$$


For calculations with a hard core in the model of boundary conditions, the right-hand side is equal to zero for $x<c$, where $c$ is the core size. To obtain an unambiguous solution to the equations, we must preset boundary conditions (7), (8),

$$
\left.\Psi_{a L}(x, y)\right|_{x=0}=0,\left.\quad \Psi_{a L}(x, y)\right|_{x=0}=0,
$$

which assume the following form in the boundary-condition model:

$$
\begin{gathered}
\Psi_{a L}(c, y)+\sum_{a^{\prime}} \int_{-1}^{+1} \Psi_{a^{\prime} L}\left(x^{\prime}, y^{\prime}\right) h_{a a^{\prime}}^{L}\left(x^{\prime}, y^{\prime}\right), \eta d \eta=0 \\
x^{\prime}=\sqrt{x^{2} / 4+3 y^{2} / 4-\sqrt{3} x y \eta / 2}, \quad y^{\prime}=\sqrt{3 x^{2} / 4+y^{2} / 4+\sqrt{3} x y \eta / 2},
\end{gathered}
$$

For $\rho \rightarrow \infty$ the boundary conditions in the case of short-range pair potentials can be written in the form $[13]$

$$
\Psi_{a L} \sim_{\rho \rightarrow \infty} a_{a L, v} \sum_{v} \psi_{l, v}(x) H_{v}\left(\sqrt{E-E_{2, l, v}}\right)+A_{a L}(\theta) \frac{\exp i \sqrt{E} \rho+i \pi L / 2}{\sqrt{\rho}}
$$

where $\psi_{l, v}(x)$ are the partial components of the wave functions of paired subsystems with binding energy $\epsilon_{l, v} ; \rho=\sqrt{x^{2}+y^{2}} ; \theta=\arctan y / x ; a_{a L, v}$ and $A_{a L}(\theta)$ are the scattering amplitudes of processes with two or three particles, respectively, in the final state; and $H_{v}(x)$ are the Hankel spherical functions.

In calculations of bound states, the wave functions decrease quite rapidly at infinity; consequently, at a large distance $x=R_{x}, y=R_{y}$, the asymptotic boundary conditions can be replaced by the conditions

$$
\begin{gathered}
\frac{\left.\partial_{x} \Psi_{a L}\right|_{x=R_{x}}}{\left.\Psi_{a L}\right|_{x=R_{x}}}=i \sqrt{\epsilon_{v}} \\
\frac{\left.\partial_{y} \Psi_{a L}\right|_{y=R_{y}}}{\left.\Psi_{a L}\right|_{y=R_{y}}}=i \sqrt{\epsilon_{v}-E}
\end{gathered}
$$

For the $\mathrm{He}_{3}$ system in the three-particle approximation with angular momentum $L=0$, we have

$$
H_{\lambda, l}=H_{0, l}=-\frac{\partial^{2}}{\partial x^{2}}-\frac{\partial^{2}}{\partial y^{2}}+l(l+1)\left(1 / x^{2}+1 / y^{2}\right)
$$

where partial components $l$ assume even values. $l=0,2,4, \ldots$; and the expression for functions $h_{a a^{\prime}}^{L}(x, y, \eta)$ is given in [13-15].

The asymptotic behavior of the components of Eqs. (9) for scattering processes with short-range potentials can be described by the function [13-15]

$$
\begin{gathered}
\Psi_{l}(x, y ; z)=\delta_{l 0} \psi_{d}(x)\left[\sin \left(\sqrt{z-\epsilon_{d}} y\right)+\exp \left(i \sqrt{z-\epsilon_{d}} y\right)\left[a_{0}(z)+o\left(y^{-1 / 2}\right)\right]\right] \\
+\frac{\exp (i \sqrt{z} \rho)}{\sqrt{\rho}}\left[A_{l}(z, \theta)+o\left(\rho^{-1 / 2}\right)\right], \quad \text { (12) }
\end{gathered}
$$

where $a_{0}(z), z=E+i 0$ is the elastic scattering amplitude for $E>\epsilon_{d}$, and $A_{l}(E, \theta)$ is the decay amplitude for $E>0$.

We also assume that the helium molecule $\mathrm{He}_{2}$ has only one bound state with binding energy $\epsilon^{d}<0$ and with the corresponding wave function $\psi_{d}(x)$.

For processes of scattering, the scattering matrix for $z=E+i 0, E>\epsilon^{d}$, the scattering phases and lengths in the $s$ state can be expressed with help of the following formulas

$$
\begin{gathered}
S_{0}(z)=1+2 i a_{0}(z), \\
\delta_{0}(p)=\frac{1}{2} I m \ln S_{0}\left(\epsilon_{d}+p^{2}+i 0\right), \quad p>0, \\
L_{s l}=-\sqrt{3} / 2 \lim _{p \rightarrow 0} a_{0}(p) / p
\end{gathered}
$$


To solve the system of equations (9) with boundary conditions (10), (12) numerically, we used the standard method described in detail in [13-15, 17]. For pair interaction potentials, we used potentials HFDHE2, HFD-B, HFDID, LM2M1, LM2M2, and TTYPT with appropriate parameters [32], which reproduce in detail the main parameters of the corresponding molecules [18].

The results of calculation of the energy of bound states in systems $\mathrm{He}_{3}$ and $\mathrm{He}_{3}^{*}$ with and without taking into account the hard core are given in Tables 1-4.

The results of calculation of the scattering states in systems $\mathrm{He}_{3}$ and $H e_{3}^{*}$ with and without taking into account the hard core are given in d Fig.4 (dependence of fase shifts on energy).

For interpreting the geometrical characteristic of the $\mathrm{He}_{3}$ molecule in both graund and exiting ststes was given in [31]. Using the method devtloped in these paper, let us consider the geometric characteristics of $\mathrm{Ne}_{3}$ and $\mathrm{Ne}_{3}^{*}$ molecules which are considerable interest in context of investigations into Bose condensation, supercondactivity and superfluidity. The results of calculation of the density function difined as [31]

$$
\varrho\left(\boldsymbol{r}_{1}\right)=\int\left|F\left(\boldsymbol{r}_{1}, \boldsymbol{r}_{2}, \boldsymbol{r}_{3}\right)\right|^{2} d \boldsymbol{r}_{2} d \boldsymbol{r}_{3},
$$

where

$$
\begin{gathered}
F\left(\boldsymbol{r}_{1}, \boldsymbol{r}_{2}, \boldsymbol{r}_{3}\right)=\Psi\left(x, y, z^{\prime}\right)+x y\left[\frac{\Psi\left(x^{+}, y^{+}, z^{\prime+}\right)}{x^{+} y^{+}}+\frac{\Psi\left(x^{-}, y^{-}, z^{\prime-}\right)}{x^{-} y^{-}}\right] / 2 \pi x y, \\
z^{\prime}=(\boldsymbol{x}, \boldsymbol{y}) / x y, \\
x^{+-}=\left(x^{2} / 4+3 y^{2} / 4-_{+} \sqrt{3} x y z^{\prime} / 2\right)^{1 / 2}, \quad y^{+-}=\left(3 x^{2} / 4+y^{2} / 4-{ }_{+} \sqrt{3} x y z^{\prime} / 2\right)^{1 / 2},
\end{gathered}
$$

are presented in Fig.2,3. This function has the form

$$
\varrho(r)=\frac{\sqrt{3}}{4 \pi^{2} r^{2}} \int\left|F\left(x, r \sqrt{3}, z^{\prime}\right)\right|^{2} d x d z^{\prime}
$$

A sufficiently clear representation of the geometrical characteristics of theinert gases is provided be plotting thes function in coordinates $r_{i}, r_{a}$, where $r_{l}=r z^{\prime} r_{a}=\frac{z^{\prime}}{\left|z^{\prime}\right|} r\left(1-z^{2}\right)^{1 / 2}$.

Note that here, for excited state of the inert gas molecules as well for the molecules $H e_{3}^{*}$, [31] and $\mathrm{Ne}_{3}^{*}$ (Fig.2,3), this function has two peaks, which corresponds to a linear structure of the $H e_{3}^{*}$ system. This corresponds to the situation when the third particle in the excited state is located with a high probability between two other particles (as if this state corresponded to two combined paired subsystems). It is precisely this configuration that corresponds to the conditions for the emergence of the Efimov effect in a three-particle system, when the scattering length in one of the paired subsystems is quite large. This conclusion is confirmed by calculations of the clusterization coefficient defined by the formula [31]

$$
f_{c}=\int \Psi\left(x, y, z^{\prime}\right) \phi_{2}(x) d z^{\prime} d x
$$

The results of such calculations are given in Table 1. It can be seen that two-particle states dominate in the excited state $\mathrm{Ne}_{3}^{*}$, while their role in the ground state is insignificant. In the ground state, system $\mathrm{He}_{3}$ forms a nearly equilateral triangle, while in the excited state, one of the atoms is at a large distance from the other two atoms. Other excited states can be obtained by the similitude method [1, 13, 15].

An analogous structure is formed in the calculation of the ground states of the systems $\mathrm{Ne}_{3}, \mathrm{Ar}_{3}$, $K r_{3}, X e_{3}$, and $R e_{3}$ using the three-particle approximation. The results of calculation of these systems in the given approximation with the HFD-B potential and the parameters borrowed from [32] are presented in Tables 5-6.

In calculations based on the boundary-condition model, the value of core $c$ was chosen so that even a slight change in this quantity did not affect the binding energy of paired subsystems. In our calculations, $c=1.5 A^{\circ}$, the value of binding energy for the helium molecule was $1.69 \mathrm{mK}$, and the value of $r_{0}$ was 100 $A^{o}$. A detailed description of the numerical method for solving system of equations (9) with asymptotic boundary conditions (11), and (12) is given in [13-15]. 
It should be noted that, according to our calculations, the size of the ground state of the $\mathrm{He}_{3}$ system is smaller than the size of the $\mathrm{He}_{2}$ molecule. However, the size of the excited state $H e_{3}^{*}$ of the three particle system is much larger than that of the two-particle system $\mathrm{He}_{2}$. The experimental data [28] confirm this statement. Thus, in the given approximation, the results of calculations indicate that peculiar resonant states can exist in the $\mathrm{He}_{3}$ system, the number of such states being not more than two.

To study the scattering processes occurring during the collision of an atom with a helium molecule and to determine the role of pair interaction potentials, we calculated the amplitudes of elastic scattering and decay as well as phase shifts with and without taking into account the hard core.

The results are almost independent of the form of pair interaction potentials and on whether or not the hard core was taken into account both for bound states and for scattering state. Thus, it can be concluded that the form of pair interaction potentials and allowance for a hard core in the boundary-condition model in the given approximation does not substantially affect the results of calculations.

Acknowledgments. The author is idebted to many people of Laboratory of Photochemistry Processes, Theoretical Department P.N.Lebedev Physical Institute, Laboratory Collision Theory Lenigrad State University who made possible his partisipation in exciting developments partialy described in this paper.

I am most grateful to Professors G.P.Drukarev, Yu.N.Demkov, S.P.Merkuriev, V.Efimov, G.J.Schulz, I.Fabricant, E.A.Soloviev, I.Yurova and many athers for initiating this study.

This study was supported by the Taiwan Academy of Sciences (grant no NSC 85-212-M-007-009), Scientific Research Foundation of the China People's Republic (grant no. NSF 19734030), Joint Research Foundation of Israel and the USA, and the Russian Foundation for Basic Research (projects nos. 98-0217266 and 01-02-16075).

\section{References}

1. V. Efimov, Influence of Resonance in Pair Forces on the Spectrum of Three-Particle Levels (Mosk. Inzh.-Fiz. Inst., Moscow, 1973) [in Russian]; Nucl. Phys. A 362, 45 (1981); Nucl. Phys. A 378, 581 (1982); Phys. Rev. C 47, 1876 (1993); S. A. Vugal'ter and G. M. Zhislin, Dokl. Akad. Nauk SSSR 267, 784 (1982).

2. T. Andras, Resonances in Few-Body Systems (Springer,New York, 2002); V. I. Kukulin, K. M. Krasnopolsky, and J. Horacek, Theory of Resonances. Principles and Applications (Kluver, Dordrecht, 1989).

3. A. Poincare, Selected Works (Nauka, Moscow, 19711972), Vols. 1-2.

4. G. J. Schultz, Rev. Mod. Phys. 45, 423 (1973).

5. G. F. Drukarev, Collisions of Electrons with Atoms and Molecules (Nauka, Moscow, 1978; Plenum, New York, 1987).

6. Yu. N. Demkov and V. N. Ostrovskii, Zero-Range Potentials and Their Applications in Atomic Physics (Leningr. Gos. Univ., Leningrad, 1975; Plenum, New York, 1988).

7. L. G. Christophorou, Electron Molecule Interaction and Their Application (Academic, New York, 1984).

8. H. S. W. Massey, Negative Ions (Cambridge Univ. Press, Cambridge, 1976; Mir, Moscow, 1979).

9. A. Herzenberg, Electron-Molecular Collision (Plenum, New York, 1984).

10. W. Domcke, Phys. Rep 208, 98 (1991); A. K. Kazanskii and I. I. Fabrikant, Usp. Fiz. Nauk 143, 601 (1984) [Sov. Phys. Usp. 27, 607 (1984)].

11. A. Chutjian, A. Garscadden, and J. M. Wadehra, Phys. Rep. 264, 393 (1996).

12. I. Prigogine and I. Strengers, Quantum, Chaos, Time. To a Solution of the Time Paradox (Editorial URSS, Moscow, 2003).

13. S. P. Merkur'ev and L. D. Faddeev, Quantum Theory of Scattering for Several Particle Systems (Nauka, Moscow, 1985) [in Russian]; D. R. Yafaev, Mathematical Theory of Scattering (S.-Peterb. Gos. Univ., St. Petersburg, 1994) [in Russian]; V. B. Belyaev, Lectures on the Theory of Small-Particle Systems (Energoizdat, Moscow, 1986) [in Russian].

14. S. A. Pozdneev, Application of Quantum Theory of Scattering to the Calculating Various Processes in Nuclear, Atomic, and Molecular Physics, in Dynamics of Elementary Atomic-Molecular Processes in Gas and Plasma, v.212, p.99-254, Nova Science Puplishers, Inc.,N.Y., 1996; Application of Quantum Theory of Scattering to the Calculating Various Processes in Nuclear, Atomic, and Molecular Physics (Yanus-K, Moscow, 2001) [in Russian].

15. S. A. Pozdneev, Phys. Lett. B 125, 335 (1983); J. Russ. Laser Res. 22, 175 (2001); in Book of Abstracts of XXI ICPEAC (Stockholm, Sweeden, 2003), p. 41; in Book of Abstracts of Heron Island International Conference on Reactive Intermediates and Unusual Molecules (Univ. of Queensland, Brisbane, Australia, 2003), p. 123. 
16. T. K. Rebane, Yad. Fiz. 61, 61 (1998) [Phys. At. Nucl.61, 56 (1998)].

17. S. A. Pozdneev, Sov. Phys. JETP 50, 18 (1979); JETP 90, 30 (2000); J. Phys. B: At. Mol. Phys. 16, 867 (1983); S. Pozdneev and G. F. Drukarev, J. Phys. B: At. Mol. Phys. 13, 2611 (1980).

18. K. P. Huber and G. Herzberg, Molecular Spectra and Molecular Structure, Vol. 4: Constants of Diatomic Molecules (Van Nostrand Reinhold, New York, 1979; Mir, Moscow, 1984).

19. H. Drexel, G. Senn, T. Fiegele, etal., J. Phys. B: At. Mol. Opt. Phys. 34, 1415 (2001).

20. A. P. Hickman, Phys. Rev. 43, 3495 (1991).

21. G. A. Gallup, Y. Xu, and I. I. Fabricant, Phys. Rev. A 57,2596 (1998); A. K. Kazansky, Y. Xu, and I. I. Fabricant,Phys. Rev. A 63, 014703 (2000); I. I. Fabricant and H. Hotop, Phys. Rev. A 63, 022706 (2001).

22. V. V. Popyshev, Yad. Fiz. 66, 64 (2003) [Phys. At. Nucl. 66, 61 (2003)]; J. Phys. A: Math. Gen. 36, L13 (2003).

23. A. V. Finkel'shtein and O. B. Ptitsin, Physics of Protein: Course of Lectures (Knizhnyi Dom Universitet, Moscow, 2002) [in Russian]; D. S. Chernavskii and N. M. Chernavskaya, Protein-machine. Biological Macromolecular Constructions (Mosk. Gos. Univ., Moscow, 1999) [in Russian]. E.A.Kaimakov, Biophysics, v.25, p.7, 1980; Preprint FTI 1735,SpB.,1999; V.F.Avetistov, V.I.Goldansky Usp.Phys.Nauk, v.166, N8, p.873,1996; V.V.Lobzin, V.R.Chechetkin Usp.Phys.Nauk, v.170, N1, p.57, 2000.

24. L. D. Landau and E. M. Lifshitz, Course of Theoretical Physics, Vol. 3: Quantum Mechanics: Non-Relativistic Theory, 4th ed. (Nauka, Moscow, 1989; Oxford Univ. Press, Oxford, 1980); B. N. Zakhar'ev and V. M. Chabanov, Controllable Quantum Mechanics. A New Status of the Theory of the Approach to Inverse Problem (Inst. Komp'yut. Issled., Moscow, 2002) [in Russian].

25. F. M. Pen'kov, JETP 91, 698 (2000); Phys. Rev. 62, 044701-1 (2000); A. K. Motovilov and F. M. Pen'kov, Selected Problems of Theoretical Physics and Astrophysics (Ob. Inst. Yad. Issled., Dubna, 2003) [in Russian].

26. Y. Horacek and W. Domke, Phys. Rev. A 53, 2262 (1996).

27. S. A. Pozdneev, JETP, v.99, N5, 2004, p.915-933.

28. F. Lon, C. F. Gliese, and W. R. Gentry, J. Chem. Phys. 104, 1151 (1996); W. Schollkopf and J. P. Toennies, J. Chem. Phys. 104, 1155 (1996); Phys. Rev. Lett. 85, 2284 (2000); M. V. Rama Krishna and K. V. Whaley, Phys. Rev. Lett. 64, 1126 (1990); G. C. Hegerfeldt and T. Kohler, Phys. Rev. Lett. 84, 3215 (2000).

29. D. V. Fedorov and A. S. Jensen, Phys. Rev. C 50, 2372,(1994); A. Cobis, Phys. Lett. B 424, 1 (1998); J. Yuan and C. D. Lin, J. Phys. B: At. Mol. Opt. Phys. 31, L637,(1998); T. Gonzalez-Lezana, J. Rubayo-Soneira, S. Miret-Aztes, et al., J. Chem. Phys. 110, 9000 (1999); Phys. Rev. Lett. 82, 1648 (1999); T. Frederico, L. Tomio, A. Delfino, et al., Phys. Rev. A 60, R9 (1999); Y. Hahn, Phys. Rev. A 60, 2139 (1999); E. Nielsen, D. V. Fedorov, and A. S. Jensen, Phys. Rev. Lett. 82, 2844 (1999); P. F. Bedaque, H. W. Hammer, and U. Van-Kolek, Phys. Rev. Lett. 82, 463 (1999); R. A. Ionescu and C. Nategan, Europhys. Lett. 45, 269 (1999).

30. A. K. Motovilov, E. A. Kalganova, and S. A. Sofianos, J. Phys. B: At. Mol. Opt. Phys. 31, 1279 (1998); J. Chem. Phys. 275, 168 (1997); Phys. Rev. A 56, R1686 (1997).

31. V. Rudnev and S. Yakovlev, Chem. Phys. Lett. 22, 97 (2000); Phys. At. Nucl. 63, 61 (2000); 63, 77 (2000); 63, 271 (2000); 63, 278 (2000); 63, 402 (2000); 63, 409 (2000); 63, 830 (2000).

32. A. R. Janzen and R. A. Aziz, J. Chem. Phys. 79, 4330 (1979); 94, 8047 (1991); 103, 9626 (1995); Mol. Phys. 61, 1487 (1987); K. T. Tang, J. P. Toenries, and C. L. Yiu, Phys. Rev. Lett. 74, 1546 (1995); S. Pozdneev, in Proceedings of Laser Chemistry, Biophysics and Biomedicine ICONO'95 (1996), p. 92; Proc. SPIE, 96 (1996). 


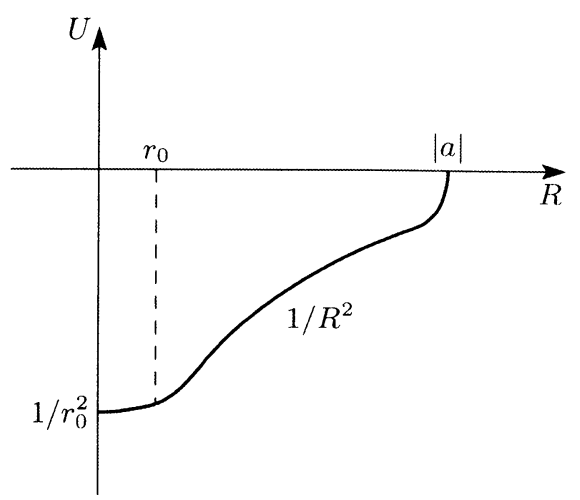

Figure 1. Effective potential responsible for resonances in a three-body system.

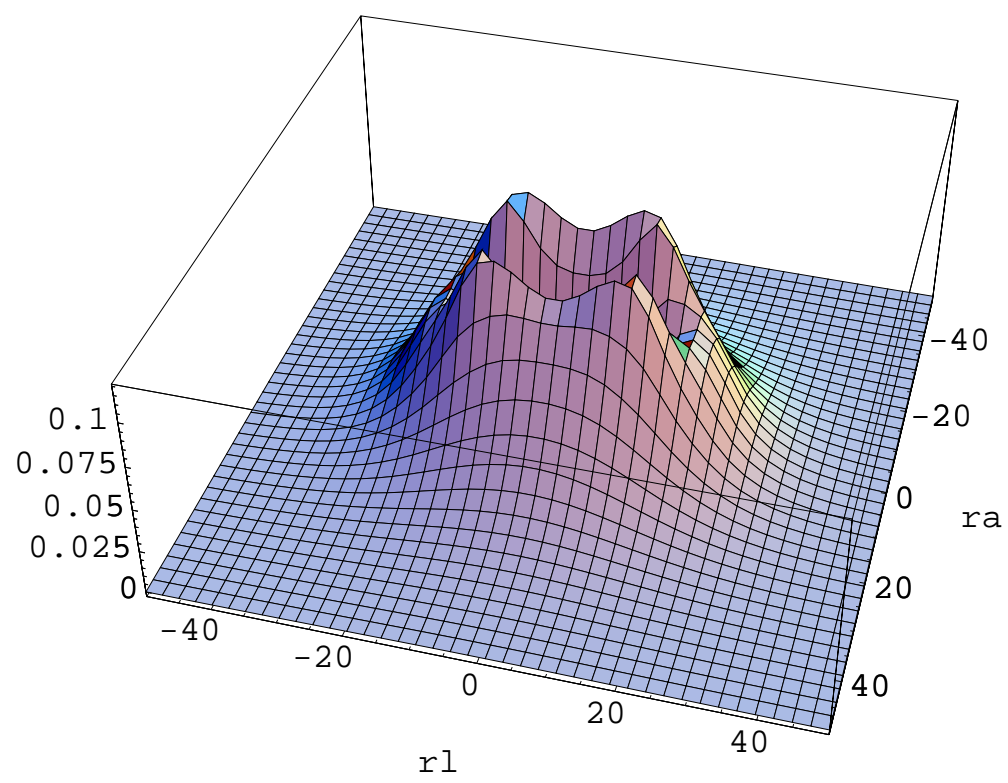

Figure 2. The density functions for $N e_{3}^{*}$ molecule in ground states. 


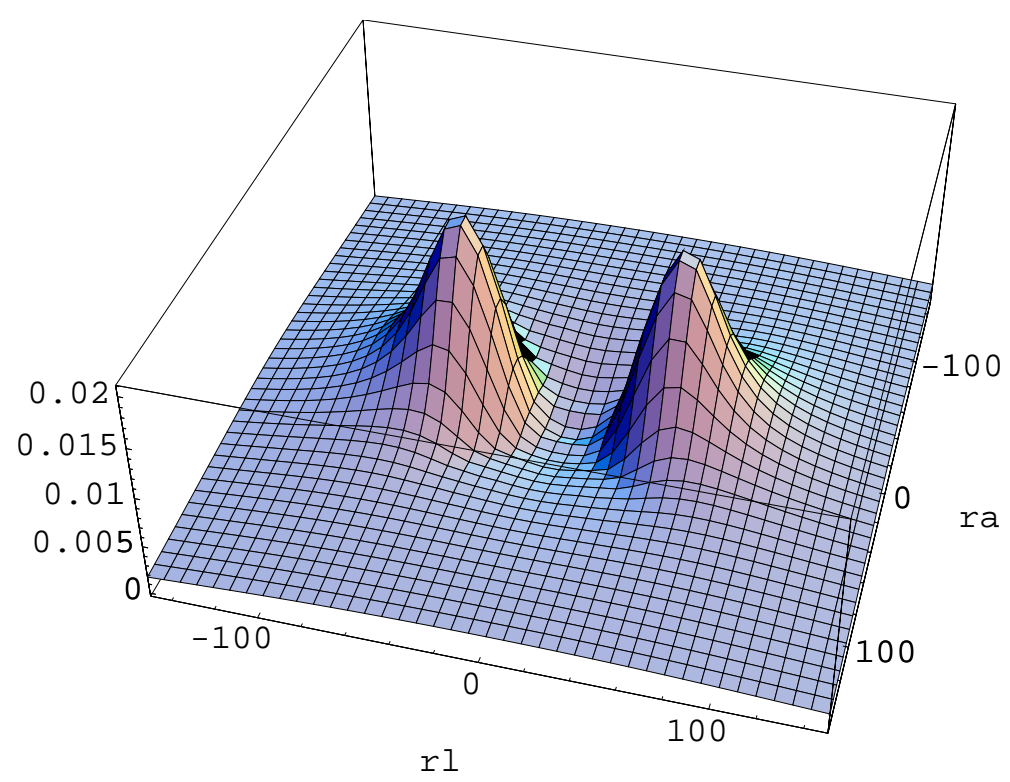

Figure 3. The density functions for $N e_{3}^{*}$ molecule in exited states. 
rhase shitts delta'

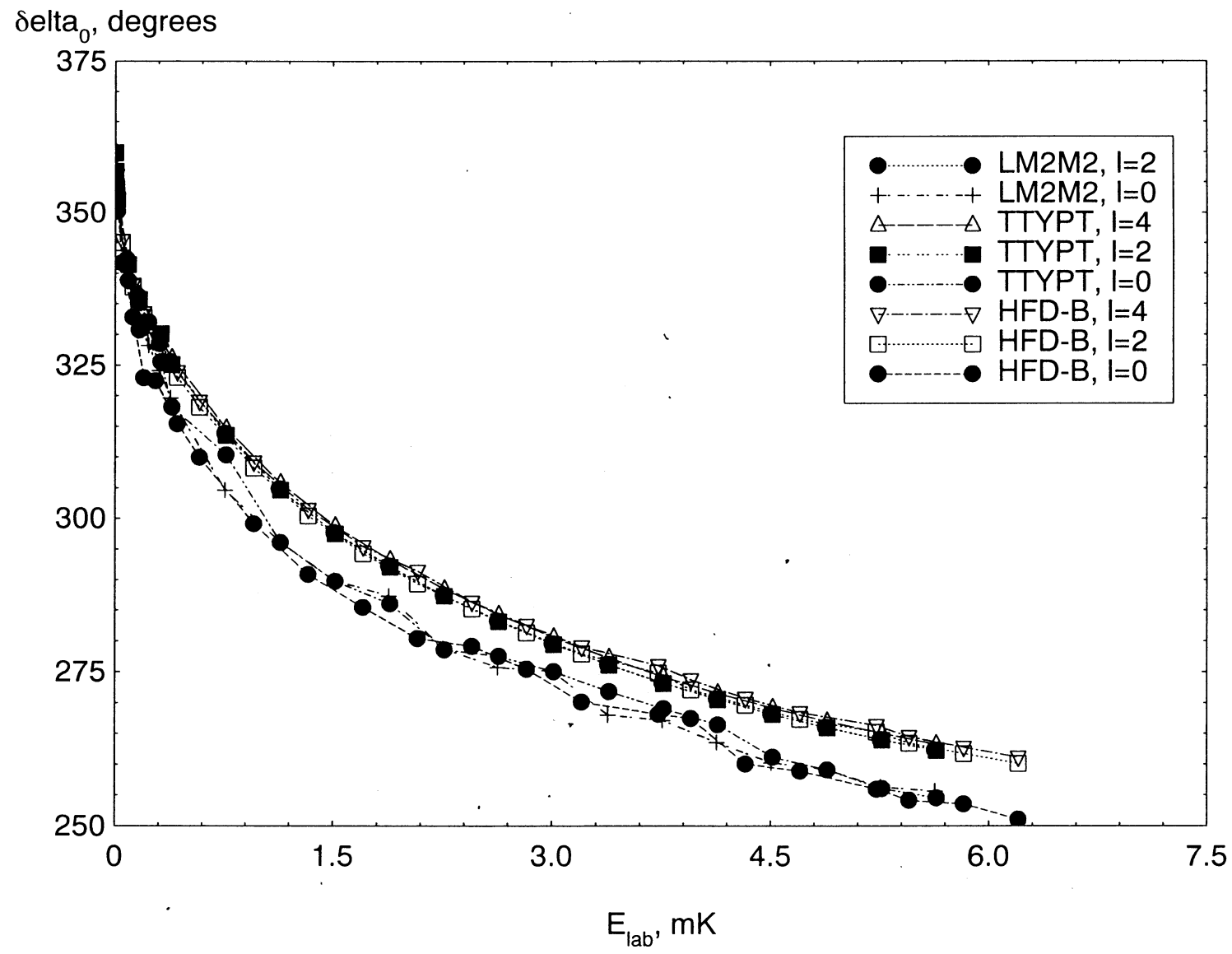

Figure 4. Dependence of fase shifts on energy for the collisions between helium atom and molecule for potentials $H F D-B, L M 2 M 2, T T Y P[32]$ calculated (a) without and (b) with taking into account the hard core.

Table 1. Binding energy, coefficient of clasterization, scattering length, mean radius and square of mean raduius for $\mathrm{He}_{3}$

\begin{tabular}{|c|c|c|c|c|c|}
\hline Potential & $E_{\mathrm{He}_{3}}, \mathrm{mK}$ & $\left\|f_{c}\right\|^{2}$ & $L_{s l}, \AA$ & $\left\langle r_{\mathrm{He}_{3}}\right\rangle, \AA$ & $\left\langle r_{\mathrm{He}_{2}}^{3}\right\rangle^{1 / 2}, \AA$ \\
\hline HFDHE2 & -0.1171 & 0.2094 & 140 & 5.65 & 6.46 \\
\hline HFD-B & -0.1330 & 0.2717 & 137 & 5.48 & 6.23 \\
\hline HFD-ID & -0.1061 & 0.1555 & 139 & 5.80 & 6.64 \\
\hline LM2M1 & -0.1247 & 0.2412 & 132 & 5.57 & 6.35 \\
\hline LM2M2 & -0.1264 & 0.2479 & 131 & 5.55 & 6.32 \\
\hline TTYPT & -0.1264 & 0.2487 & 130 & 5.56 & 6.33 \\
\hline
\end{tabular}


Table 2. Binding energy, coefficient of clasterization, scattering length, mean radius and square of mean raduius for $\mathrm{He}_{3}$ in boundary-condition model

\begin{tabular}{|c|c|c|c|c|c|}
\hline Potential & $E_{\mathrm{He}_{3}}, \mathrm{mK}$ & $\left\|f_{c}\right\|^{2}$ & $L_{s l}, \AA$ & $\left\langle r_{\mathrm{He}_{3}}\right\rangle, \AA$ & $\left\langle r_{\mathrm{He}_{2}}^{3}\right\rangle^{1 / 2}, \AA$ \\
\hline HFDHE2 & -0.1170 & 0.2095 & 138 & 5.65 & 6.46 \\
\hline HFD-B & -0.1329 & 0.2717 & 135 & 5.48 & 6.23 \\
\hline HFD-ID & -0.10612 & 0.1555 & 134 & 5.80 & 6.64 \\
\hline LM2M1 & -0.12465 & 0.2412 & 130 & 5.57 & 6.35 \\
\hline LM2M2 & -0.12641 & 0.2479 & 131 & 5.55 & 6.32 \\
\hline TTYPT & -0.12640 & 0.2487 & 131 & 5.56 & 6.33 \\
\hline
\end{tabular}

Table 3. Binding energy, coefficient of clasterization, scattering length, mean radius and square of mean raduius for $\mathrm{He}_{3}^{*}$

\begin{tabular}{|c|c|c|c|c|c|}
\hline Potential & $E_{H e_{3}^{*}}, \mathrm{mK}$ & $\left\|f_{c}^{*}\right\|^{2}$ & $L_{s l}, \AA$ & $\left\langle r_{H e_{3}^{*}}\right\rangle, \AA$ & $\left\langle r_{H e_{3}^{*}}^{3}\right\rangle^{1 / 2}, \AA$ \\
\hline HFDHE2 & -1.6653 & 0.9077 & 134 & 55.26 & 66.25 \\
\hline HFD-B & -2.743 & 0.9432 & 135 & 48.33 & 57.89 \\
\hline HFD-ID & -1.0612 & 0.8537 & 140 & 62.75 & 75.38 \\
\hline LM2M1 & -2.1550 & 0.9283 & 129 & 51.53 & 61.74 \\
\hline LM2M2 & -2.2713 & 0.9319 & 131 & 50.79 & 60.85 \\
\hline TTYPT & -2.2806 & 0.9323 & 131 & 50.76 & 60.81 \\
\hline
\end{tabular}

Table 4. Binding energy, coefficient of clasterization, scattering length, mean radius and square of mean raduius for $\mathrm{He}_{3}^{*}$ in boundary condition model

\begin{tabular}{|c|c|c|c|c|c|}
\hline Potential & $E_{H e_{3}^{*}}, \mathrm{mK}$ & $\left\|f_{c}^{*}\right\|^{2}$ & $L_{s l}, \AA$ & $\left\langle r_{H e_{3}^{*}}\right\rangle, \AA$ & $\left\langle r_{H e_{3}^{*}}^{3}\right\rangle^{1 / 2}, \AA$ \\
\hline HFDHE2 & -1.6765 & 0.9078 & 135 & 56.22 & 67.11 \\
\hline HFD-B & -2.7458 & 0.9439 & 135 & 48.31 & 58.00 \\
\hline HFD-ID & -1.1061 & 0.8597 & 136 & 62.87 & 76.13 \\
\hline LM2M1 & -2.2585 & 0.9323 & 132 & 52.41 & 62.04 \\
\hline LM2M2 & -2.2801 & 0.9319 & 131 & 50.79 & 61.05 \\
\hline TTYPT & -2.2885 & 0.9339 & 131 & 51.23 & 60.89 \\
\hline
\end{tabular}

Table 5. Binding energies of inert gas molecules calculated by using HFD-B potential, a.u. $10^{-6}$

\begin{tabular}{|c|c|c|c|c|c|}
\hline Energy & $N e_{2}$ & $A r_{2}$ & $K r_{2}$ & $X e_{2}$ & $R n_{2}$ \\
\hline$E_{t h r}$ & 178 & 394 & 619 & 854 & 9268 \\
\hline$E_{\text {exp }}$ & 135 & 446 & 629 & 874 & - \\
\hline
\end{tabular}


Table 6. Binding energies of the ground state and the first excited state of the inert gas molecules calculated by using HFD-B potential

\begin{tabular}{|c|c|c|c|c|c|c|c|c|c|}
\hline$N e_{3}$ & $N e_{3}^{*}$ & $A r_{3}$ & $A r_{3}^{*}$ & $K r_{3}$ & $K r_{3}^{*}$ & $X e_{3}$ & $X e_{3}^{*}$ & $R n_{3}$ & $R n_{3}^{*}$ \\
\hline 398 & 330 & 1278 & 1215 & 1885 & 1811 & 2509 & 2438 & 30875 & 30801 \\
\hline
\end{tabular}

